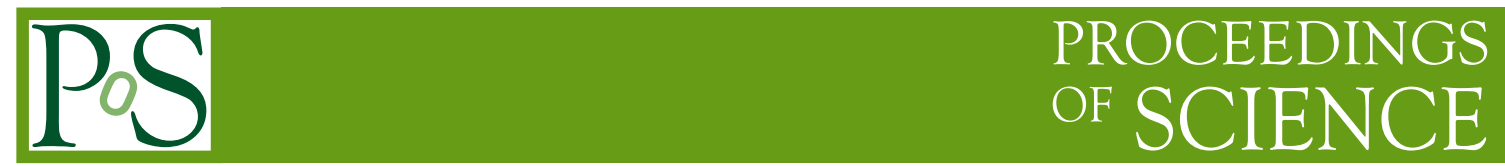

\title{
The ArgoNeuT Experiment
}

\section{J. Spitz, for the ArgoNeuT Collaboration}

Department of Physics, Yale University, New Haven, CT 06520, USA

E-mail: joshua.spitz@yale.edu

\begin{abstract}
ArgoNeuT is a Liquid Argon Time Projection Chamber neutrino experiment that recently completed its physics run in the NuMI beamline at Fermilab. Along with research and design for future LArTPCs, the experiments goals include performing a number of neutrino and anti-neutrino cross section measurements. Also, ArgoNeuT hopes to further the understanding of the nuclear physics involved in neutrino scattering by characterizing the low energy protons created in such interactions.
\end{abstract}

35th International Conference of High Energy Physics - ICHEP2010,

July 22-28, 2010

Paris France

${ }^{*}$ Speaker. 


\section{Introducing the LArTPC}

A Liquid Argon Time Projection Chamber (LArTPC) [1] design is an attractive candidate for a kiloton-scale long baseline neutrino oscillation experiment far detector. Such a device would also double as a detector with an eye towards proton decay, atmospheric neutrino oscillation, supernova burst/diffuse neutrino detection, and more. The purpose of any neutrino detector is to detect and reconstruct those particles that were created in a neutrino-nucleon (or -electron, -nucleus) interaction in order to infer properties of the neutrino, such as flavor and energy. In a LArTPC, the interaction products emanating from the interaction vertex ionize argon atoms as they traverse the detector, leaving a trail of ionization in their wake. In an ultra-pure environment, these trails are free to drift across the noble liquid medium. An electric field is imposed in the detector and the trails are drifted towards two or more sensing wire planes that are oriented at an angle with respect to one another. Combining the wire plane and timing information provides a full three dimensional image of the event. Also, as the charge induced and collected on the wire planes is proportional to the amount of energy deposited by the particle along its track, calorimetric information is obtained as well. Argon, with a relatively large density, short radiation length, and high scintillation yield, is ideal for neutrino detection and event containment.

Although LArTPCs present a multitude of advantages over other neutrino detection methods, there are a number of technical challenges associated with their design and operation. Achieving and maintaining a liquid purity sufficient to drift the ionization trails with little or no attenuation over a few meters is quite difficult. Other challenges include mitigating the potential oxygen deficiency hazard of a large argon spill (possibly underground), routing very high voltage inside the detector, light collection techniques, maximizing signal-to-noise, keeping the argon cold, detector construction underground, and more. Developing an automated neutrino reconstruction software is also a major challenge.

\section{ArgoNeuT}

The ArgoNeuT (NSF/DOE) experiment's physics run in the NuMI beamline at Fermilab lasted from September 2009 to February 2010. During this time, ArgoNeuT collected the first $>10,000$ neutrino and anti-neutrino events ever with a LArTPC in a low energy neutrino beam ${ }^{1}$. ArgoNeuT was located just upstream of the on-axis MINOS [3] near detector and collected about 1.35E20 protons on target (POT) in the "low energy" NuMI configuration (0.1E20 POT in neutrino mode and 1.25E20 POT in anti-neutrino mode). The $175 \mathrm{~L}$ active volume TPC $\left(47 \times 40 \times 90 \mathrm{~cm}^{3}\right)$ featuring two instrumented wire planes (480 channels total, with a wire spacing of $4 \mathrm{~mm}$ and $5 \mathrm{MHz}$ sampling frequency) can be seen in Figure 1. ArgoNeuT is an important R\&D step in the US HEP phased program towards a massive kiloton-scale LArTPC. Along with cross section measurements, ArgoNeuT also hopes to demonstrate the particle identification capabilities of LArTPCs. Electron/gamma separation is especially relevant for future electron-neutrino appearance experiments searching for non-zero $\theta_{13}$ and $\delta_{C P}$. An example of a neutrino event in ArgoNeuT involving

\footnotetext{
${ }^{1}$ Note that the ICARUS 50 L [2] detector in the CERN West Area Neutrino Facility was the first LArTPC in a neutrino beam.
} 
several gammas can be seen in Figure 2. ArgoNeuT is also heavily involved in developing LArTPC reconstruction techniques in collaboration with the MicroBooNE [4] and LBNE experiments.

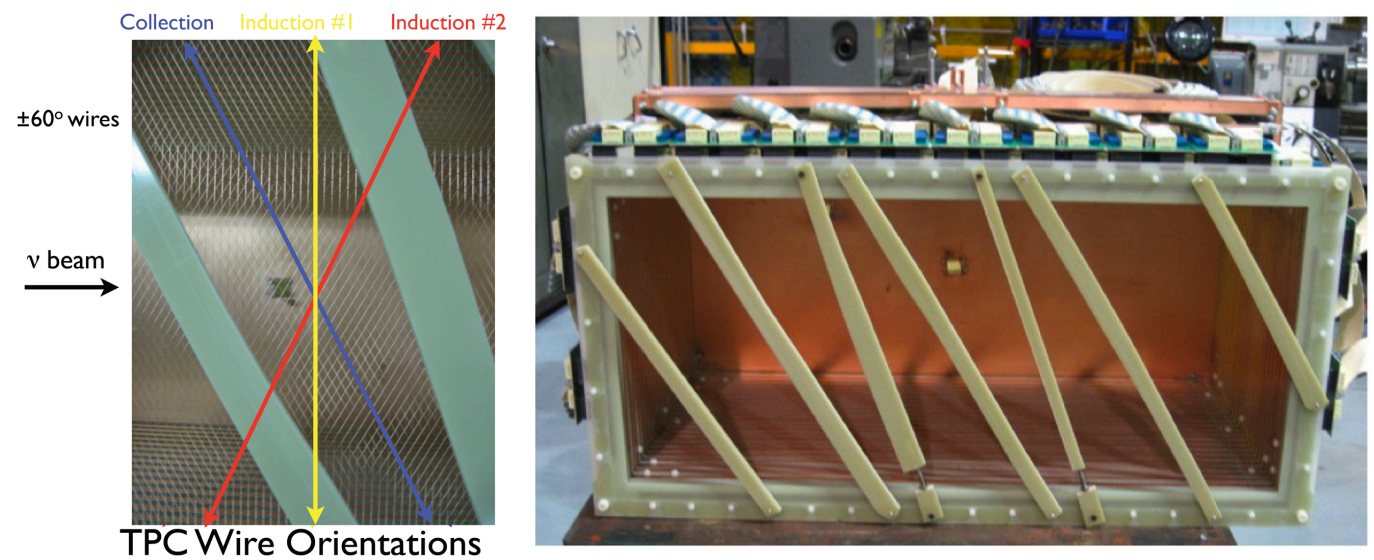

Figure 1: Left: The orientation of the ArgoNeuT TPC's wire planes. Note that only the "Induction \#2" and "Collection" plane are instrumented. Right: The fully assembled TPC.

\section{The CCQE Interaction and Vertex Activity}

With only an outgoing lepton and nucleon (before final state interactions), a Charged Current, Quasi-Elastic $\left(\mathrm{CCQE}^{2}\right)$ event is perhaps the easiest (anti-)neutrino interaction to identify and reconstruct. Neutrino flavor is unambiguously found by determining the type of lepton exiting the nucleus. For $v_{\mu}$ events, calorimetric reconstruction is done quite simply due to the "single-track" nature (as opposed to a more difficult to reconstruct electron- or photon-induced multi-track electromagnetic shower) of the outgoing particles. Also, the CCQE cross section is comparatively high in the energy region of interest to long baseline neutrino oscillation experiments. For these reasons, the CCQE channel is considered the "signal" channel for such experiments. Despite its importance in long baseline neutrino oscillation physics, the CCQE cross section is known with only 20-30\% precision over most of the relevant energy range $(\sim 0.5-10 \mathrm{GeV})$. Furthermore, the experimental definition of "CCQE" varies across experiments that have more or less sensitivity to the hadrons that exit the nucleus (see, for example, Ref [5] and [6]). A consistent picture of the "vertex activity", or low energy hadrons exiting the nucleus, associated with CCQE events is needed in order to account for such differences.

With the assistance of the downstream magnetized MINOS near detector's muon sign selection capabilities, ArgoNeuT has the unique ability to compare neutrino and anti-neutrino events in terms of vertex activity. Such a comparison may be able to shed light on a possible CCQE multinucleon channel [7] in which, before final state interactions, 2 protons (neutrons) are emitted in a CCQE neutrino (anti-neutrino) event. ArgoNeuT's CCQE analysis currently centers on describing the vertex activity associated with such events, a vital step towards an ultimate CCQE differential/absolute cross section measurement.

\footnotetext{
${ }^{2}$ For neutrinos, $v_{l} n \longrightarrow l^{-} p$. For anti-neutrinos, $\bar{v}_{l} p \longrightarrow l^{+} n$.
} 

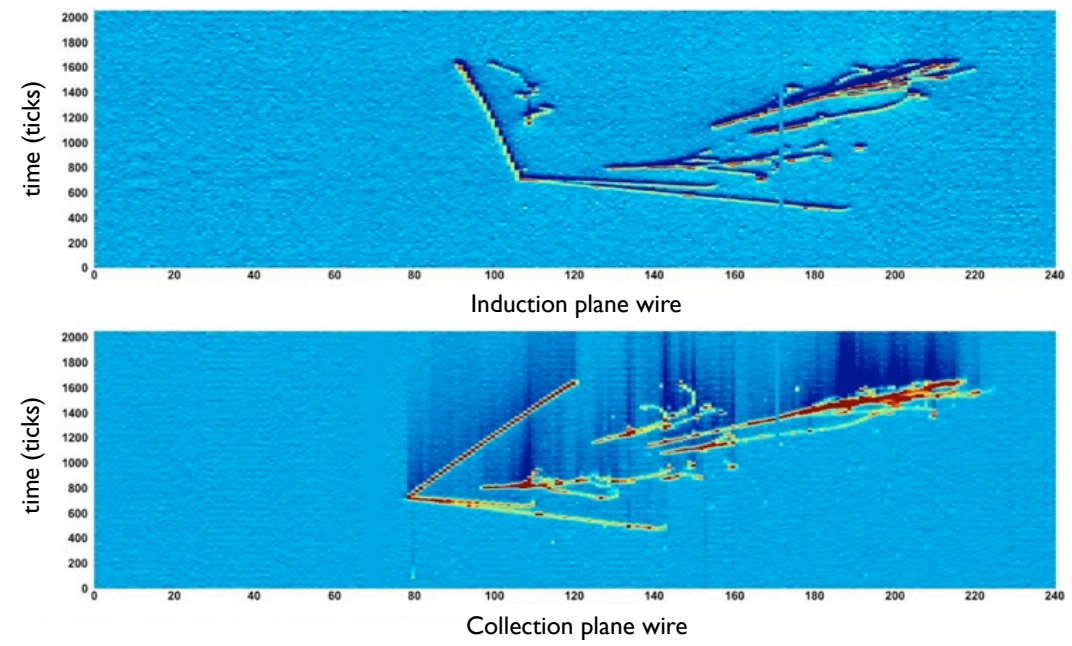

Figure 2: A deep inelastic scattering neutrino event in ArgoNeuT as seen in the induction and collection plane views. The four electromagnetic showers with displaced vertices indicate that two $\pi^{0}$ s were created in the neutrino interaction (which decayed quickly after creation, $\pi^{0} \rightarrow \gamma \gamma$ ).

\section{Conclusion}

ArgoNeuT recently collected over 10,000 neutrino and anti-neutrino events during its 5 month physics run. The interactions are currently being analyzed in an attempt to perform neutrino cross section and vertex activity measurements. ArgoNeuT is developing an automated neutrino reconstruction software to be used for these analyses and future LArTPC-based experiments. The successful ArgoNeuT construction and stable physics run also represent a major step forward towards the technical realization of a kiloton-scale LArTPC.

\section{References}

[1] C. Rubbia, The Liquid Argon Time Projection Chamber: a New Concept for Neutrino Detector, CERN-EP/77-08 (1977).

[2] F. Arneodo, et al. Performance of a liquid argon time projection chamber exposed to the CERN West Area Neutrino Facility neutrino beam, Phys. Rev. D 74 (2006) 112001 [physics / 0609205 ].

[3] D. Michael, et al. Observation of muon neutrino disappearance with the MINOS detectors and the NuMI neutrino beam, Phys. Rev. Lett. 97 (2006) 191801 [hep-ex/0607088].

[4] H. Chen, et al. Proposal for a New Experiment Using the Booster and NuMI Neutrino Beamlines: MicroBooNE, FERMILAB-PROPOSAL-0974 (2007).

[5] A. A. Aguilar-Arevalo, et al. First Measurement of the Muon Neutrino Charged Current Quasielastic Double Differential Cross Section, Phys. Rev. D 81 (2010) 092005 [hep-ex/10 02 . 2680].

[6] V. Lyubushkin, et al. A study of quasi-elastic muon neutrino and antineutrino scattering in the NOMAD experiment, Eur. Phys. J. C 63 (2009) 255 [hep-ex/ 0812 . 4543].

[7] M. Martini, et al.Unified approach for nucleon knock-out and coherent and incoherent pion production in neutrino interactions with nuclei, Phys. Rev. C 80 (2009) 065501 [nucl-th/0910.2622]. 